\title{
Nanoscale hepatoprotective herbal decoction attenuates hepatic stellate cell activity and chloroform-induced liver damage in mice
}

This article was published in the following Dove Press journal:

International Journal of Nanomedicine

30 June 2011

Number of times this article has been viewed

\author{
Sherry Huang' \\ Shu-Jen Chang ${ }^{2}$ \\ Miffy Yang ${ }^{3}$ \\ Justin Jin-Ching Chen ${ }^{3}$ \\ Walter $\mathrm{H} \mathrm{Chang}^{4}$ \\ 'Department of Biomedical \\ Engineering, Chung Yuan Christian \\ University, Chungli, Taiwan; ${ }^{2}$ School \\ of Pharmacy, China Medical University, \\ Taichung, Taiwan; ${ }^{3}$ Center for Nano \\ Bioengineering, ${ }^{4}$ Department of \\ Biomedical Engineering, Chung Yuan \\ Christian University, Chungli, Taiwan
}

Correspondence: Walter $\mathrm{H}$ Chang Department of Biomedical Engineering, Chung Yuan Christian University,

Chungli 32023, Taiwan

Tel +88632654503

Fax +8863265458 I

Email whchang@cycu.edu.tw
Background: San-Huang-Xie-Xin-Tang (SHXXT) decoction, a traditional Chinese medicine containing Rhei rhizome, Coptidis rhizome, and Scutellariae radix, is widely used in hepatoprotective therapy. However, preparation of the decoction requires addition of boiling water that causes loss of numerous effective components.

Methods: To improve the bioavailability of the decoction, nanoscale SHXXT was developed. Chloroform-induced liver injury and hepatic stellate cell activity in mice were used to demonstrate the hepatoprotective characteristics of nanoscale SHXXT decoction.

Results: Liver/body weight ratio and serum aspartate and alanine aminotranferase levels were recovered by the nanoscale SHXXT. TIMP-1 gene expression was inhibited and MMP-2 gene expression was accelerated in activated hepatic stellate cells.

Conclusion: Nanoscale SHXXT decoction prepared in room temperature water could have preserved hepatoprotective ability. The results of this study indicate that nanoscale SHXXT could be extracted easily. The simple preparation of this herbal decoction is more convenient and energy-efficient.

Keywords: Chinese herb medicine, San-Huang-Xie-Xin-Tang, nanoparticle, liver fibrosis

\section{Introduction}

There has been a tremendous wave of interest in the mechanism of liver fibrosis in the last few decades. A growing number of research studies are shedding light on the relationship between liver fibrosis and herbal medicine therapy. Liver fibrosis is a dynamic and reversible wound healing process of chronic liver injury that tends to result in cirrhosis and hepatoma. Liver fibrosis is usually caused by viral hepatitis infection, alcohol abuse, or nonalcoholic steatohepatitis. The fibrotic process involves excessive deposition of extracellular matrix in liver tissue. ${ }^{1,2}$ San-Huang-Xie-Xin-Tang (SHXXT) decoction is a traditional hepatoprotective Chinese herbal medicine, comprising Rhei rhizome (rhizomes of Rheum officinale Baill.), Scutellariae radix (roots of Scutellaria baicalensis Georgi.), and Coptidis rhizoma (rhizomes of Coptis chinesis Franch.) at a ratio of $2: 1: 1 .{ }^{3}$ SHXXT decoction is widely used in anti-inflammatory ${ }^{4,5}$ and antioxidant ${ }^{3}$ preparations. SHXXT decoction can also regulate the expression of genes involved in p53 signaling, p53 activation, and DNA damage signaling pathways in hepatocytes. Furthermore, the regulation of SHXXT may be associated with antiproliferation patterns. ${ }^{6}$

As with most Chinese herbal medicines, SHXXT decoction is extracted in boiling water to release the biological components. However, poor solubility decreases bioavailability, so considerable quantities of these herbal drugs must be used..$^{7-9}$ 
Increasing the dissolution rate of hydrophobic drugs is the key to overcoming these obstacles. According to the Noyes-Whitney equation, decreased particle size gives rise to increased drug surface area and dissolution velocity. ${ }^{10-12}$ Due to the larger surface area of nanoparticles per mass unit, researchers have proposed that these are more biologically active than bulky particles with the same constituent. Nanoscale Chinese herbal drugs can typically be defined as drug systems with particles in the size range of $10-1000 \mathrm{~nm} .^{13}$

To evaluate the effect of nanoscale SHXXT decoction on injured livers, chloroform was used in this study to cause liver damage in mice. Chloroform can increase the liver/body weight ratio and the cell nuclear antigen-labeling index. ${ }^{14}$ Cytochrome P450-dependent chloroform metabolism is lost during repeated chloroform treatments in $\mathrm{B} 6 \mathrm{C} 3 \mathrm{~F} 1$ mice. ${ }^{15}$ Chloroform-induced liver injury in rodents is an effective animal model for the assessment of drug therapy. ${ }^{16}$ One of the major sources of extracellular matrix proteins that contribute to the process of liver fibrosis is the hepatic stellate cell. During liver fibrogenesis, an increase in hepatic stellate cell proliferation and upregulation of extracellular matrix proteins is typical. A transformation growth factor-beta 1 (TGF- $\beta 1$ )-activated hepatic stellate cell culture model has been used in several studies. ${ }^{17-21}$

This study investigated the hepatoprotective ability of a nanoscale SHXXT decoction prepared with room temperature water. Microscale SHXXT decoction prepared with boiling water was used as the comparator group. Two models, in vivo and in vitro, were examined in this study. Female imprinting control region mice were exposed to chloroform twice a week to induce liver damage, after which the nanoscale SHXXT decoction prepared with room temperature water was applied to cure the mice. Liver/body weight ratio, serum aspartate aminotransferase (AST), alanine aminotranferase (ALT), and histological features of the liver were investigated. In the cell culture assay, TGF- $\beta 1$ was applied to induce hepatic stellate cell activity, and then treated with nanoscale SHXXT decoction or microscale SHXXT decoction. TMTT activity, and MMP-2 and TIMP-1 mRNA expression were determined. The intention of this study was to contrast the two methods and to determine if the nanoscale SHXXT decoction prepared with room temperature water has hepatoprotective activity.

\section{Material and methods}

\section{Preparation of nanoscale SHXXT}

Chinese herbal SHXXT containing Rhei rhizome, Scutellariae radix, and Coptidis rhizome was purchased from the China Medical University. Dried mixtures of Rhei rhizome/
Scutellariae radix/Coptidis rhizome (2:1:1 by weight) were nanoscaled with the aid of a pulsed air-flow pulverizer (Diamond Nano-Biochem Company, Taiwan). Briefly, the herbal mixture was ground into small pieces $(3 \mathrm{~mm})$. Afterwards, the small pieces were placed into the chamber of the pulsed air-flow pulverizer. The airflow provided the collision between the herbal mixtures. Thus, the particle size of the herbal mixture was attenuated to nanoscale. The powders were stored at room temperature until analysis.

\section{Measurement of hydrodynamic diameters of nanoscale SHXXT}

The hydrodynamic diameters of nanoscale SHXXT were examined by dynamic laser light scattering in the single scattering regime with $\lambda=532 \mathrm{~nm}$ (Zeta Sizer Nano ZS; Malvern Instruments Ltd, Worcestershire, UK). Typically, nanoscale SHXXT was suspended in Milli-Q water $(1.5 \mathrm{~mL})$ using ultrasonic vibration at a concentration of $0.1 \mathrm{mg} / \mathrm{mL}$. The suspension was put into a cuvette at $25^{\circ} \pm 1^{\circ} \mathrm{C}$ to enable analysis of particle size. The viscosity and refractive index of the continuous phase were set to those specific for water.

\section{Field emission scanning electron microscopy}

To demonstrate the morphology of nanoscale SHXXT, the samples were examined by field emission scanning electron microscopy (S-4100; Hitachi, Tokyo, Japan) operating at $15 \mathrm{kV}$. The samples used were prepared by suspending $1 \mathrm{mg}$ of nanoscale SHXXT in $1 \mathrm{~mL}$ of Milli-Q water. The suspended nanoparticles were pipetted in $200 \mu \mathrm{L}$ amounts onto the holder, and dehydrated. To enhance electrical conductivity, the samples were coated with platinum via sputtering (SPI-Module Sputter Coater; SPI Supplies - Structure Probe, Inc, West Chester, PA) before examination.

\section{Preparation of nanoscale SHXXT decoction}

Nanoscale SHXXT ( $200 \mathrm{~g}$ ) was extracted with $1 \mathrm{~L}$ of room temperature Milli-Q water, whereas microscale SHXXT (200 g) was extracted with $1 \mathrm{~L}$ of boiling Milli-Q water. The aqueous extract, called nanoscale SHXXT decoction and microscale SHXXT decoction, was separated from the nanoscale SHXXT particles and microscale SHXXT particles by filters and then used for the experiment.

\section{Experimental animals}

Laboratory-bred, 6-8 week-old imprinting control region mice sourced from the National Taiwan University Hospital, 
Taipei, Taiwan, were used in this study. The animals were housed at $21^{\circ}-25^{\circ} \mathrm{C}$, a relative humidity of $30 \%-70 \%$ and on a 12-hour/12-hour day/night cycle. Pelleted mouse feed (MF Laboratory Animal Diet, Oriental Yeast, Tokyo, Japan) and reverse-osmosis water were provided ad libitum. All procedures used in this study were approved by the institutional animal ethics committee.

\section{Animal treatment}

To investigate the protective effects of nanoscale SHXXT decoction against liver injury, the mice were divided into four groups: a control group, which was neither administered SHXXT decoction nor exposed to chloroform; a chloroform group, which was administered chloroform but was not exposed to SHXXT decoction; a microscale SHXXT decoction treatment group, which was administered the microscale SHXXT decoction and chloroform; and a nanoscale SHXXT decoction treatment group, which was administered the nanoscale SHXXT decoction and chloroform. SHXXT was administered via the intragastric route at a dose of $2.9 \mathrm{~mL} / \mathrm{kg}$ per day for 8 weeks. In the same way, chloroform was intragastrically administered at a dose of $0.2 \mathrm{~mL} / \mathrm{kg}$ twice per week for 8 weeks. Each group included eight mice. At the end of the experiment, all the mice were sacrificed, and their livers and blood were collected immediately. Liver weights were subsequently measured.

\section{AST and ALT measurement}

Blood was obtained from the vena cava before autopsy, and the serum was isolated and stored at $-80^{\circ} \mathrm{C}$ until analysis. Serum AST and ALT levels were estimated using commercial kits (Human Diagnostics, Wiesbaden, Germany) according to the manufacturer's protocols.

\section{Histological analysis}

Liver tissue was isolated, fixed in $10 \%$ buffered neutral formalin, and processed for paraffin embedding. Thereafter, the tissue was cut into $5 \mu \mathrm{m}$ thin sections and stained with hematoxylin and eosin solution. Hepatic morphology was assessed by light microscopy.

\section{Cell culture}

The hepatic stellate cells used in this study were gifted from Professor Friedman (Division of Liver Diseases, Mount Sinai School of Medicine, New York). The cells were grown to confluence in a T-75 flask (Falcon, Heidelberg, Germany) in a Waymouth MB 752/1 medium supplemented with $10 \%$ fetal bovine serum at $37^{\circ} \mathrm{C}$ in a $5 \% \mathrm{CO}_{2}$ atmosphere.

\section{Detection of hepatic stellate cell proliferation}

Hepatic stellate cell proliferation was measured using a colorimetric MTT [3-(4,5-dimethylthiazolyl-2)-2, 5-diphenyltetrazolium bromide] assay. After 7 days of culture, the hepatic stellate cells were transferred into flat-bottomed 96-well microtiter plates at a seeding density of $2 \times 10^{3}$ cells/well, and were cultured again for 24 hours. The culture medium was removed and replaced by Waymouth MB 752/1 medium containing the microscale SHXXT decoction or the nanoscale SHXXT decoction. After 48 hours, the culture medium was removed again and replaced by Waymouth MB 752/1 medium containing MTT. The cells were then incubated for another 4 hours prior to the MTT assays, which were then performed using a Spectra Max 190 plate reader (Molecular Devices, Toronto, ON). The results were obtained at $570 \mathrm{~nm}$. The optical density values of groups treated with microscale SHXXT decoction and nanoscale SHXXT decoction were compared with the TGF- $\beta 1$ induced only group. Each experiment was performed eight times in each group.

\section{Reverse-transcription polymerase chain reaction analysis}

The hepatic stellate cells were seeded in six-well plates at a seeding density of 200,000 cells/well and treated with $2 \mathrm{ng} / \mathrm{mL}$ TGF- $\beta 1$ and $500 \mu \mathrm{g} / \mathrm{mL}$ microscale SHXXT decoction or nanoscale SHXXT decoction for 24 hours. Total RNA was isolated using the Geneaid kit (Geneaid, Taipei, Taiwan) according to the manufacturer's instructions. First-strand complementary DNA was synthesized from $5.0 \mu \mathrm{g}$ total RNA per $30 \mu \mathrm{L}$ reaction volume. The polymerase chain reaction was performed using the following primers: rat MMP-2 (604 bp), 5'-GTGCTGAAGGACACCCTCAAGAAGA-3' (sense) and 5'-TTGCCGTCCTTCTCAAAGTTGTACG-3' (antisense), rat TIMP-1 (310 bp), 5'-GCCATGGAGAGCCTCTGTGG-3' (sense) and 5'-GCAGGCAGGCAAAGTGATCG-3' (antisense), and rat GAPDH (74 bp), 5'-AACTACATGGTCTACATGTTCCA-3' (sense) and 5'-CCATTCTCGGCCTTGACTGT-3' (antisense). All reactions were performed in a total volume of $30 \mu \mathrm{L}$ containing $3 \mu \mathrm{L}$ $10 \times$ polymerase chain reaction buffer, $0.5 \mu \mathrm{L} 5 \mathrm{U}$ Taq DNA polymerase, $1 \mu \mathrm{L} 10 \mathrm{mM}$ dNTP, $2 \mu \mathrm{L}$ target primer mixture, and $1 \mu \mathrm{L}$ cDNA as a template. The products of polymerase chain reaction were analyzed using $2 \%$ agarose gels and 
ethidium bromide staining. The products were then visualized under ultraviolet light and saved digitally with a UVIdoc gel documentation system (Uvitec, Cambridge, UK).

\section{Statistical analysis}

Data were expressed as mean \pm standard error values and analyzed statistically using one-way analysis of variance followed by Scheffé's test using SPSS (v 14.0; SPSS, Inc, Chicago, IL) to establish the significance of any differences. The level of statistical significance was set at $P<0.05$.

\section{Results and discussion \\ Particle size distribution of nanoscale SHXXT}

The size and shape of the nanoscale SHXXT were determined by zeta sizer and field emission scanning electron microscopy. The measurements were performed by dispersing the nanoscale SHXXT in Milli-Q water using ultrasonic vibration. From the dynamic laser light scattering data presented in Figure 1A, the mean diameters of nanoscale SHXXT were determined to be approximately $600 \mathrm{~nm}$. The field emission scanning electron photomicrograph of the nanoscale SHXXT particles shown in Figure 1B illustrates the oblong shape and confirms the size of the particles to be similar to the zeta size results. These data indicate that the dried SHXXT was successfully nanoscaled by pulsed airflow pulverizer. The pulsed air-flow pulverizer was applied to the herbal nanoparticles ${ }^{22-24}$ and calcium nanoparticle ${ }^{25}$ preparations.

\section{Changes in ratio of liver/body weight by nanoscale SHXXT decoction}

The ratio of liver/body weight was increased by oral administration of chloroform (Figure 2A). Chloroform caused dose-dependent increases in liver/body weight ratios. ${ }^{14}$ Chloroform is toxic to the liver. Furthermore, mice receiving the microscale SHXXT decoction and the nanoscale SHXXT decoction had a lower liver/body weight ratio during the chloroform treatment period (Figure $2 \mathrm{~A}$ ). These results suggest that the nanoscale SHXXT decoction reduced the chloroform-induced liver/body weight ratio gain.

\section{Changes in serum ALT and AST}

ALT and AST are often used as markers for evaluating liver function. ${ }^{26,27}$ In the present study, ALT and AST activity was

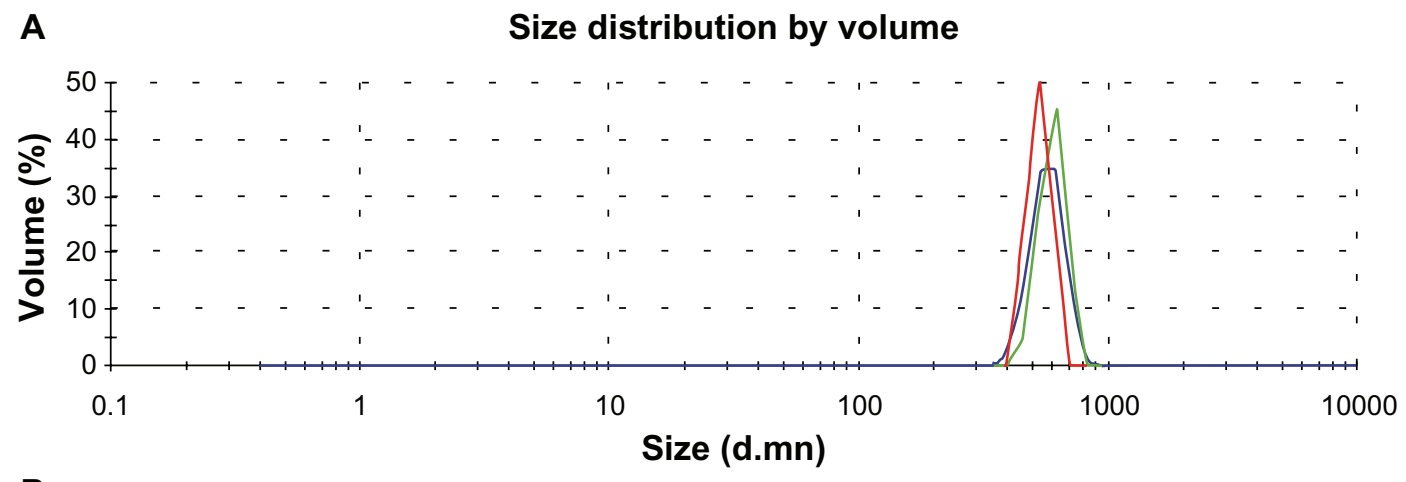

B

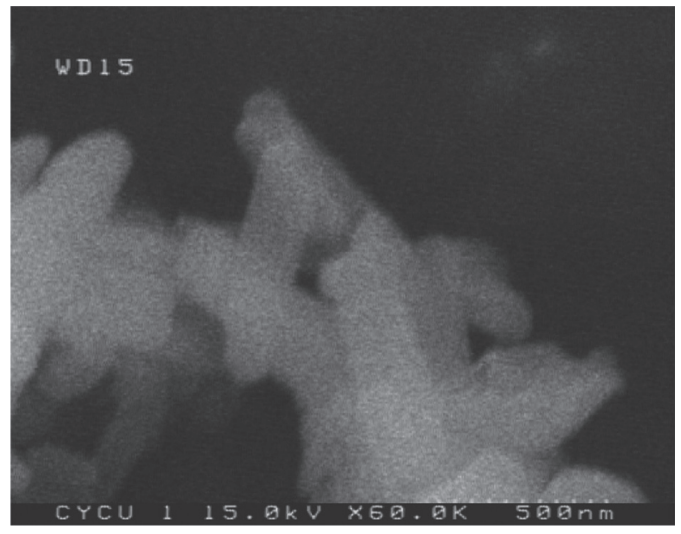

Figure I Size and shape of the nanoscale SHXXT decoction. A) Zeta sizer picture of nanoscale SHXXT showing size distribution in Milli-Q water. B) Field emission scanning electron microscopy picture of nanoscale SHXXT on a solid substrate.

Abbreviation: SHXXT, San-Huang-Xie-Xin-Tang. 

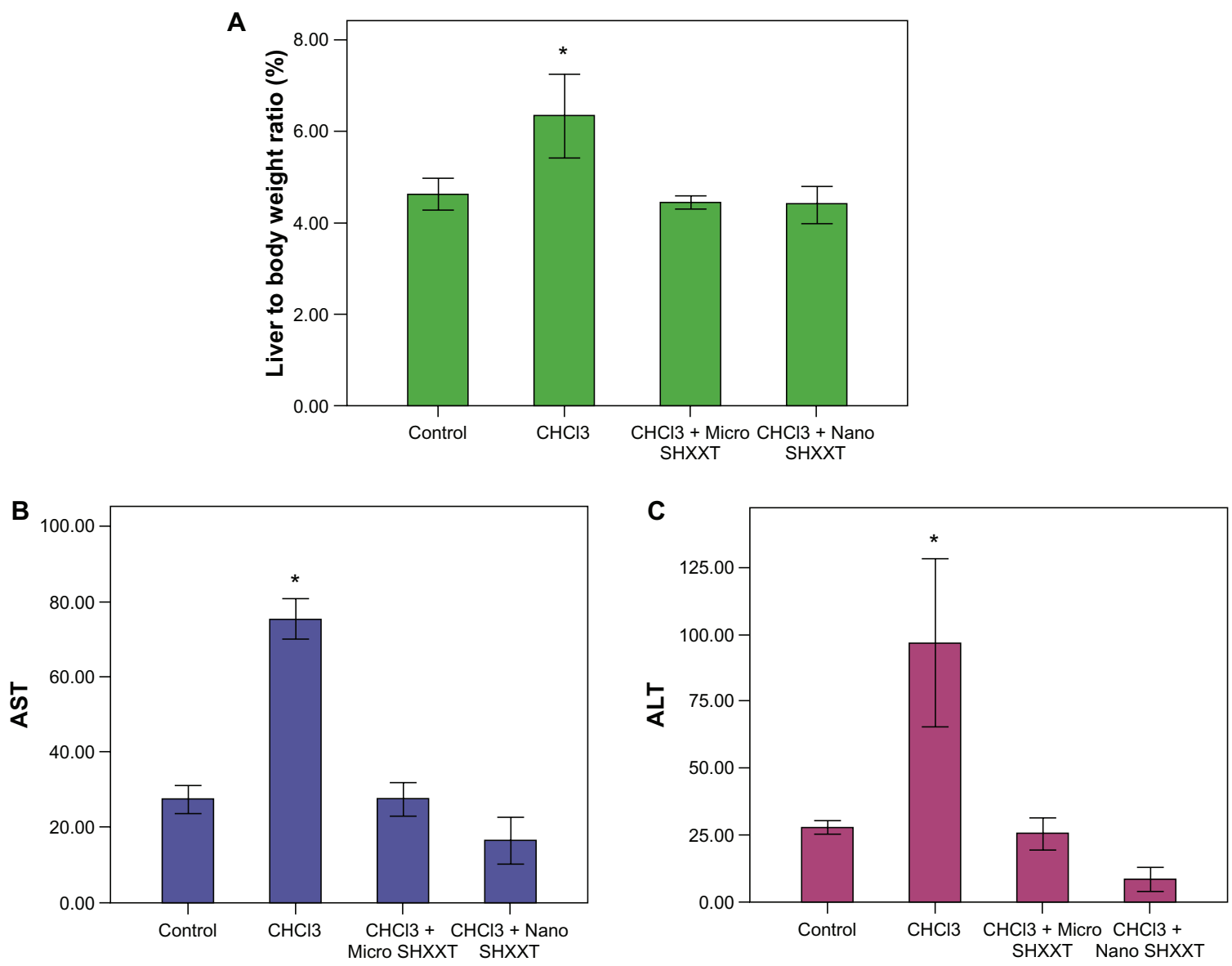

Figure 2 Effects of chloroform, microscale SHXXT decoction, and nanoscale SHXXT decoction on A) liver-to-body-weight ratio, B) AST level, and C) ALT level. Notes: Results are represented as means \pm standard error of the mean. *Significant difference from control $(P<0.05)$.

Abbreviations: SHXXT, San-Huang-Xie-Xin-Tang; ALT, alanine aminotransferase; AST, aspartate aminotransferase.

significantly increased after chloroform exposure $(P<0.05)$ as compared with controls (Figures 2B and 2C). Administration of the microscale SHXXT decoction and the nanoscale SHXXT decoction decreased the chloroform-induced increase in ALT and AST activity. Treatment with the microscale SHXXT decoction and the nanoscale SHXXT decoction $(2.9 \mathrm{~mL} / \mathrm{kg}$ body weight) reversed the liver damage caused by chloroform.

\section{Histological examination}

In the sections of control liver tissue, the hepatocytes are arranged in strands with one or two spherical nuclei, and the sinusoids were occupied by blood cells. The cytoplasm of the hepatic cells was slightly eosinophilic, and the central vein generally had a circular outline (Figure 3A). Chloroform produced hepatic necrosis, inflammation, fatty accumulation, and fibrosis after 8 weeks (Figure 3B). Necrosis, inflammation, fatty accumulation, and fibrosis were significantly lower in the groups treated with the microscale SHXXT decoction and the nanoscale SHXXT decoction when compared with the chloroform-treated groups (Figures 3C and 3D). Histopathological evaluation was performed twice for four sections per slide from all animals in each group.
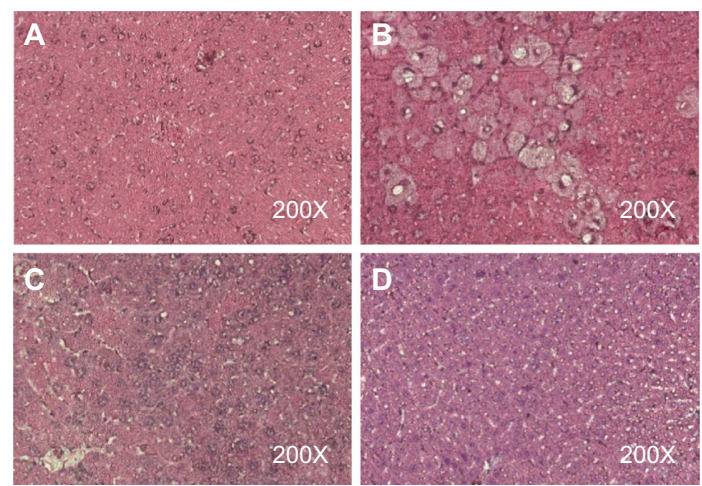

Figure 3 Liver tissue sections of A) control group, B) chloroform group, C) microscale SHXXT decoction group, and D) nanoscale SHXXT decoction group stained with hematoxylin and eosin.

Abbreviation: SHXXT, San-Huang-Xie-Xin-Tang. 
A

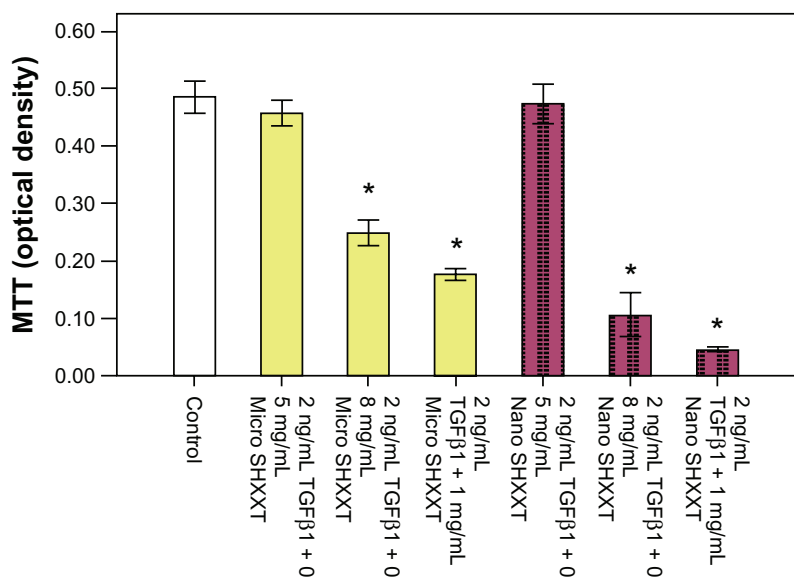

B

GAPDH

MMP-2

TIMP-1

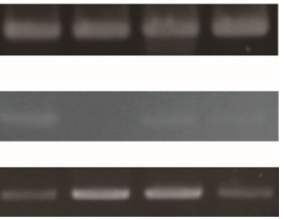

Figure 4 Effects of nanoscale SHXXT decoction on A) cell proliferation and viability of hepatic stellate cells as determined by MTT assay. Concentration-dependent cytotoxic effects were evaluated after 48 hours of incubation. Results are represented as means \pm standard error of the mean. B) Expression of GAPDH, MMP-2, and TIMP-I mRNA in hepatic stellate cells. Line I, control group; line 2, TGF- $\beta$ I; line 3, TGF $\beta$ I + microscale SHXXT decoction; line 4, TGF- $\beta$ I + nanoscale SHXXT decoction.

Notes: *Significant difference from control $(P<0.05)$.

Abbreviations: TGF- $\beta$ I, transforming growth factor-betal; SHXXT, San-Huang-Xie-Xin-Tang.

\section{MTT assay}

The results of the MTT assay demonstrated that hepatic stellate cells exposed to the nanoscale SHXXT decoction for 48 hours resulted in dose-dependent inhibition. At $0.8 \mathrm{mg} / \mathrm{mL}$ and $1 \mathrm{mg} / \mathrm{mL}$ concentrations of the microscale SHXXT decoction and nanoscale SHXXT decoction, the viability of hepatic stellate cells was reduced significantly (Figure 4A). Viability in the nanoscale SHXXT decoction groups was more efficient than in the microscale SHXXT decoction groups.

\section{Reverse transcription polymerase chain reaction}

Results from the polymerase chain reaction analysis show the changes in mRNA levels of fibrogenesis-related genes (Figure 4B). TGF- $\beta 1$ is an activation factor in hepatic stellate cells. The expression of TIPM-1, a fibrogenesis-related gene, was upregulated by TGF- $\beta 1$. Expression of MMP-2, an antifibrogenesis-related gene, was downregulated by TGF- $\beta 1$. However, the nanoscale SHXXT decoction and the nanoscale SHXXT decoction attenuated the activity of TGF- $\beta 1$ in hepatic stellate cells. The nanoscale SHXXT decoction exhibited more efficiency than the nanoscale SHXXT decoction.

\section{Conclusion}

According to this study, the pulsed air-flow pulverizer can produce nanoscale Chinese herbal medicine efficiently. This method could help us to improve the bioavailability of traditional Chinese herbal medicine. The herbal decoction can be prepared using room temperature water rather than boiling water. The pharmaceutical activity of the nanoscale SHXXT decoction was retained. The results of this study indicate that nanoscale SHXXT particles can be extracted easily. Simple preparation of a herbal decoction using this method is more convenient and energy-efficient.

Downregulation of the liver/body weight ratio and a decrease in AST and ALT levels in chloroform-induced liver injury demonstrated the excellent recovery ability of the nanoscale SHXXT decoction. The inhibition of TIMP-1 gene expression and acceleration of MMP-2 gene expression in the activated hepatic stellate cells suggests that the nanoscale SHXXT decoction may block the development and progression of liver fibrosis by altering matrix degradation. However, further studies are required to clarify the pathophysiologic role of this nanoscale SHXXT decoction in the process of liver fibrosis and cirrhosis.

\section{Acknowledgment}

This work was supported by grants from the Committee on Chinese Medicine and Pharmacy, Department of Health, Executive Yuan, Taiwan.

\section{Disclosure}

The authors report no conflicts of interest in this work.

\section{References}

1. Friedman SL. Mechanisms of disease: Mechanisms of hepatic fibrosis and therapeutic implications. Nat Clin Pract Gastr. 2004;1(2):98-105.

2. Friedman SL. Evolving challenges in hepatic fibrosis. Nat Rev Gastroenterol Hepatol. 2010;7(8):425-436.

3. Lo YC, Tsai PL, Huang YB, et al. San-Huang-Xie-Xin-Tang reduces lipopolysaccharide-induced hypotension and inflammatory mediators. J Ethnopharmacol. 2005;96(1-2):99-106. 
4. Wang YS, Lin RT, Cheng HY, Yang SF, Chou WW, Juo SH. Anti-atherogenic effect of San-Huang-Xie-Xin-Tang, a traditional Chinese medicine, in cultured human aortic smooth muscle cells. J Ethnopharmacol. 2011;133(2):442-447.

5. Shih YT, Wu DC, Liu CM, Yang YC, Chen IJ, Lo YC. San-HuangXie-Xin-Tang inhibits Helicobacter pylori-induced inflammation in human gastric epithelial AGS cells. J Ethnopharmacol. 2007;112(3): 537-544.

6. Cheng WY, Wu SL, Hsiang CY, et al. Relationship between San-HuangXie-Xin-Tang and its herbal components on the gene expression profiles in HepG2 cells. Am J Chin Med. 2008;36(4):783-797.

7. Liu M, Li YG, Chou GX, Cheng XM, Zhang M, Wang ZT. Extraction and ultra-performance liquid chromatography of hydrophilic and lipophilic bioactive components in a Chinese herb Radix Salviae Miltiorrhizae. J Chromatogr A. 2007;1157(1-2):51-55.

8. Zuo XJ, Okada Y, Toyoda M, et al. Hydrophobic extracts of a Chinese herb (CMX-13) exhibit potent immunosuppressive properties and prevent acute rejection in a highly histoincompatible model of rat lung transplantation. Transplantation. 2000;70(7):1094-1098.

9. Yap HK, Zuo XJ, Toyoda M, et al. Immunosuppressive effect of the hydrophobic extract of a Chinese herb on rat lung allograft rejection. Transplant Proc. 1998;30(4):980-981.

10. Tinke AP, Vanhoutte K, De Maesschalck R, Verheyen S, De Winter H. A new approach in the prediction of the dissolution behavior of suspended particles by means of their particle size distribution. J Pharm Biomed Anal. 2005;39(5):900-907.

11. Rinaki E, Dokoumetzidis A, Macheras P. The mean dissolution time depends on the dose/solubility ratio. Pharm Res. 2003;20(3): 406-408.

12. Jinno J, Kamada N, Miyake M, et al. Effect of particle size reduction on dissolution and oral absorption of a poorly water-soluble drug, cilostazol, in beagle dogs. J Control Release. 2006;111(1-2):56-64.

13. Huang S, Chang WH. Advantages of nanotechnology-based Chinese herb drugs on biological activities. Curr Drug Metab. 2009;10(8): 905-913.

14. Coffin JC, Ge RR, Yang SM, Kramer PM, Tao LH, Pereira MA. Effect of trihalomethanes on cell proliferation and DNA methylation in female B6C3F1 mouse liver. Toxicol Sci. 2000;58(2):243-252.

15. Rossi S, Gemma S, Fabrizi L, Testai E, Vittozzi L. Time dependence of chloroform-induced metabolic alterations in the liver and kidney of B6C3F1 mice. Arch Toxicol. 1999;73(7):387-393.
16. Golden RJ, Holm SE, Robinson DE, Julkunen PH, Reese EA Chloroform mode of action: Implications for cancer risk assessment. Regul Toxicol Pharmacol. 1997;26(2):142-155.

17. Bolkenius U, Hahn D, Gressner AM, Breitkopf K, Dooley S, Wickert L. Glucocorticoids decrease the bioavailability of TGF-beta which leads to a reduced TGF-beta signaling in hepatic stellate cells. Biochem Biophys Res Commun. 2004;325(4):1264-1270.

18. Ikeda H, Yatomi Y, Yanase M, et al. Effects of lysophosphatidic acid on proliferation of stellate cells and hepatocytes in culture. Biochem Biophys Res Commun. 1998;248(2):436-440.

19. Jung YA, Lee KM, Kim MK, et al. Forkhead transcription factor FoxO1 inhibits insulin- and transforming growth factor-beta-stimulated plasminogen activator inhibitor-1 expression. Biochem Biophys Res Commun. 2009;386(4):757-761.

20. Yang KL, Chang WT, Chuang CC, Hung KC, Li EIC. Antagonizing TGF-beta induced liver fibrosis by a retinoic acid derivative through regulation of ROS and calcium influx. Biochem Biophys Res Commun. 2008;365(3):484-489.

21. Zhao CY, Chen W, Yang L, Chen LH, Stimpson SA, Diehl AM. PPAR gamma agonists prevent TGF beta $1 \mathrm{Smad} 3$-signaling in human hepatic stellate cells. Biochem Biophys Res Commun. 2006;350(2): 385-391.

22. Ma PY, Fu ZY, Su YL, Ma JJ. The nano pulverization of traditional Chinese medicine Liuwei Dihuang. JWuhan Univ Technol. 2006;21(2): $105-108$.

23. Su YL, Fu ZY, Quan CJ, Wang WM. Fabrication of nano Rhizama Chuanxiong particles and determination of tetramethylpyrazine. Trans Nonferrous Met Soc China. 2006;16(1):S393-S397.

24. Liu JR, Chen GF, Shih HN, Kuo PC. Enhanced antioxidant bioactivity of Salvia miltiorrhiza (Danshen) products prepared using nanotechnology. Phytomedicine. 2008;15(1-2):23-30.

25. Huang S, Chen JC, Hsu CW, Chang WH. Effects of nano calcium carbonate and nano calcium citrate on toxicity in ICR mice and on bone mineral density in an ovariectomized mice model. Nanotechnology. 2009;20(37):375102.

26. Nyblom H, Berggren U, Balldin J, Olsson R. High AST/ALT ratio may indicate advanced alcoholic liver disease rather than heavy drinking. Alcohol Alcohol. 2004;39(4):336-339.

27. Giannini E, Botta F, Fasoli A, et al. Progressive liver functional impairment is associated with an increase in AST ALT ratio. Digest Dis Sci. 1999;44(6):1249-1253.
International Journal of Nanomedicine

\section{Publish your work in this journal}

The International Journal of Nanomedicine is an international, peerreviewed journal focusing on the application of nanotechnology in diagnostics, therapeutics, and drug delivery systems throughout the biomedical field. This journal is indexed on PubMed Central,

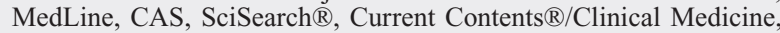

\section{Dovepress}

Journal Citation Reports/Science Edition, EMBase, Scopus and the Elsevier Bibliographic databases. The manuscript management system is completely online and includes a very quick and fair peer-review system, which is all easy to use. Visit http://www.dovepress.com/ testimonials.php to read real quotes from published authors. 